\title{
Frontières
}

\section{Christian Chelebourg, Les Écofictions. Mythologies de la fin du monde, Bruxelles, Les impressions nouvelles, coll. « Réflexions faites », 2012, 254 p.}

\section{Patrick Bergeron}

Volume 25, numéro 2, 2013

URI : https://id.erudit.org/iderudit/1024946ar

DOI : https://doi.org/10.7202/1024946ar

Aller au sommaire du numéro

Éditeur(s)

Université du Québec à Montréal

ISSN

1916-0976 (numérique)

Découvrir la revue

Citer ce compte rendu

Bergeron, P. (2013). Compte rendu de [Christian Chelebourg, Les Écofictions.

Mythologies de la fin du monde, Bruxelles, Les impressions nouvelles, coll.

" Réflexions faites ", 2012, 254 p.] Frontières, 25(2), 143-145.

https://doi.org/10.7202/1024946ar d'utilisation que vous pouvez consulter en ligne.

https://apropos.erudit.org/fr/usagers/politique-dutilisation/ 
CANGUILHEM, Georges (1998 [1965]). La connaissance de la vie, Paris, J. Vrin.

GARLAND-THOMSON, Rosemarie (2004). «Du prodige à l'erreur : les monstres de I'Antiquité à nos jours», dans N. BANCEL, P. BLANCHARD, G. BOËTSCH, É. DEROO, et S. LEMAIRE (dir.), Zoos humains: au temps des exhibitions humaines, Paris, La Découverte/poche.

GUILHAUMOU, Jacques (2005). «Les monstres dans l'ordre social: généalogies du "monstre en politique" de Machiavel à Sieyès», dans R. Bertrand et A. Carol (dir.), Le monstre humain: imaginaire et société, Aix-en-Provence, Publications de I'Université de Provence, p. 179-190.

\section{Christian Chelebourg \\ Les Écofictions. Mythologies de la fin du monde}

Bruxelles, Les impressions nouvelles, coll. «Réflexions faites», 2012, 254 p.

\section{Patrick Bergeron}

Professeur agrégé, Département d'études françaises, Université du Nouveau-Brunswick

«Au calendrier de I'Apocalypse, écrit Christian Chelebourg, une date chasse l'autre. » Celle du 21 décembre 2012, qui correspond à la fin du $13^{\text {e }}$ baktun (cycle long) dans le calendrier maya, s'ajoute ainsi à une liste déjà longue de dates faussement fatidiques. On sait maintenant que I'humanité a encore du temps devant elle. Toutefois, l'idée selon laquelle la Terre et I'Homo sapiens sont en péril ne cesse depuis quelques années de refluer dans l'imaginaire collectif. On n'a qu'à se rappeler les récents films de Roland Emmerich et de Lars von Trier, 2012 (2009) et Melancholia (2011). Si, techniquement parlant, la fin du monde n'a pas encore eu lieu, elle est constamment reconduite dans le domaine de la fiction (surtout dans la littérature et au cinéma). C'est de ce phénomène de société que rend compte Christian Chelebourg en prenant appui sur une vaste sélection de romans, de films, de bandes dessinées, de documentaires, d'essais et de publicités. Ceux-ci ont en commun d'exploiter les nouvelles peurs qui secouent jour après jour les sociétés industrielles: pollution, réchauffement climatique, effet de serre, perforation de la couche d'ozone, désastres naturels, épidémies, parmi d'autres fléaux contemporains. Tantôt, d'anciens mythes sont réactivés, tantôt de nouveaux sont créés. Les signes de l'apocalypse sont donc partout. Un demi-siècle après le grand classique de Roland Barthes, Chelebourg propose à son tour une étude de nos «mythologies». C'est d'ailleurs le sous-titre qu'il a donné à son ouvrage: Mythologies de la fin du monde.

Professeur à I'Université de Lorraine où il dirige l'équipe d'accueil «Littérature, Imaginaire, Sociétés» (anciennement Centre d'études littéraires Jean Mourot), Christian Chelebourg est spécialiste de littérature française du XIX siècle (Mérimée, Hugo, Verne), d'imaginaire et de fiction pour la jeunesse, sujet de son plus récent livre (Les fictions de jeunesse, PUF, 2013). 


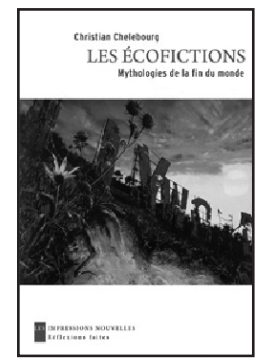

Orné en couverture d'une magnifique (mais sombre) illustration d'Alexis Rockman, artiste américain participant à une mouvance que Francesca Gavin qualifie de «nouvel art gothique», Les Écofictions vient combler une lacune dans le domaine des scienceshumaines: hormis les études à thématique restreinte (par exemple celles qui portent sur les films catastrophe ou sur les histoires de zombies), très peu d'ouvrages ont en effet été consacrés aux fictions apocalyptiques. Celles-ci pullulent pourtant depuis la fin du XXe siècle. Il s'agit même d'un sous-genre de science-fiction solidement établi depuis que maints auteurs de renom, tels Wells, Rosny aîné, London, Barjavel et Ballard, ont proposé de magistrales variations sur le motif de la fin du monde.

Chelebourg a forgé le néologisme d'écofictions pour désigner «les produits [du] nouveau régime de médiatisation des thèses environnementalistes», dont le champ ne se limite pas aux œuvres de fiction, mais recouvre aussi l'ensemble des discours qui ont recours à l'invention narrative afin de répandre le message écologique. L'écofiction ne correspond pas à une étiquette générique, applicable à des œuvres littéraires ou cinématographiques, mais à «une manière d'entrer en résonnance avec l'imaginaire d'une époque fascinée par sa puissance et terrifiée par un avenir dans lequel elle ne sait plus lire que des promesses de déclin». Déclin, mais pas forcément déclinisme: les écofictions permettent à «l'homme écofictionnel», qui se trouve en leur centre, d'affirmer un sentiment de grandeur (quoique de manière ambivalente: I'humanité est capable de ravager aussi bien que de sauver la planète) et de satisfaire un désir d'involution (le fantasme d'un retour à un âge d'or mythifié). La mythification est un processus déterminant ici, car les écofictions, qui s'articulent autour d'un rapport patrimonial à la planète, impliquent une réappropriation et une réinterprétation du patrimoine mythologique de I'humanité.

Même s'il fait appel à un apocalyptisme qui jouit d'une longue tradition dans I'Occident christianisé - par exemple, la rêverie sur les ruines du présent plonge ses racines dans le gothique anglais du XVIII ${ }^{\mathrm{e}}$ siècle - , le concept d'écofiction renvoie à un contexte récent, que Chelebourg situe après la chute du mur de Berlin. Dans les années 1990, I'alarmisme écologique a pris le relais de la rivalité Est-Ouest, devenue obsolète au sortir de la Guerre froide. L'homme écofictionnel, qui se pense en voie de disparition et évoque en ce sens I'Homo disparitus d'Alan Weisman, évolue dans un contexte de fictionnalisation des données scientifiques, telles les hypothèses du climatologue James Hansen sur le réchauffement climatique ou celles du paléo-écologiste Anthony D. Barnosky sur la possibilité d'une sixième extinction de masse.

On ne trouve ni jargon ni argumentation aride dans Les Écofictions. Le propos de Chelebourg est dense et truffé de références à des contenus spécifiques (une gamme impressionnante de textes et de films, tous cités en bibliographie), ce qui aurait pu compliquer la lecture si l'auteur 
n'avait pas pris soin d'équilibrer les cinq chapitres de son ouvrage et de les agrémenter de précieux intertitres. On saura gré à l'auteur d'intégrer à son propos des pointes d'humour, comme lorsqu'il évoque, au sujet du jeu vidéo Resident Evil, les écofictions «casse-zombies», héritières de «la logique récréative du casse-briques». On retiendra surtout l'efficacité heuristique de ce travail. L'analyse fondée sur un corpus à ce point mixte et étendu apparaît comme le meilleur moyen de traiter un sujet qui, finalement, confine à l'anthropologie culturelle et à la mythopoétique. Le concept d'écofiction, on peut en être sûr, fera des petits.

\section{CHRISTIE, Deborah et LAURO, Sarah Juliet (dir.)}

\section{Better Off Dead. The Evolution of Zombie as Post-Human}

New York, Fordham University Press, 2011, 296 p.

\section{Patrick Bergeron}

Professeur agrégé, Département d'études françaises, Université du Nouveau-Brunswick

N'en doutons plus: il existe bel et bien un champ d'«études zombie». Non seulement des associations telles que la Zombie Research Society et la Zombie Science: Zombie Institute for Theoretical Studies ont-elles vu le jour, mais les travaux universitaires sur le sujet se multiplient depuis peu. L'engouement savant pour ce revenant anthropophage atteint de telles proportions que le vampire trouve en lui un sérieux rival. Better off dead exploite ainsi une thématique dans I'air du temps. Même le domaine francophone, pourtant moins porté vers les déambulateurs d'outretombe, ajoute une pierre à l'édifice avec les ouvrages récents de Maxime Coulombe (Petite philosophie du zombie, PUF, 2012) et d'Antonio Domínguez Leiva (Invasion zombie, Murmure, 2013).

Comme le signalent les deux directrices scientifiques en ouverture du livre, il est ironique que Better off dead paraisse alors qu'on venait de commémorer le bicentenaire de Darwin et le $150^{\mathrm{e}}$ anniversaire de L'origine des espèces. Le zombie correspondrait-il à un nouveau stade évolutif au cours duquel l'Histoire continuerait de s'écrire mais sans I'homme? Pourquoi pas: après tout, l'imagination a tous les droits. Or, malgré le caractère factice du zombie, I'hypothèse d'une post-humanité n'appartient plus seulement à la science-fiction, ainsi qu'en témoignent les travaux de chercheurs comme Jean-Michel Besnier, Rosi Braidotti, Dominique Lecourt et Cary Wolfe.

Deborah Christie est professeure adjointe à I'université ECPI à Virginia Beach et Sarah Juliet Lauro, professeure adjointe invitée à I'université Clemson en Caroline du Sud. Aidées de treize collaborateurs, elles ont entrepris d'élucider l'omniprésence du zombie dans la culture populaire à partir d'une approche interdisciplinaire attentive aux origines folkloriques et cinématographiques du monstre. Il n'est donc pas seulement 